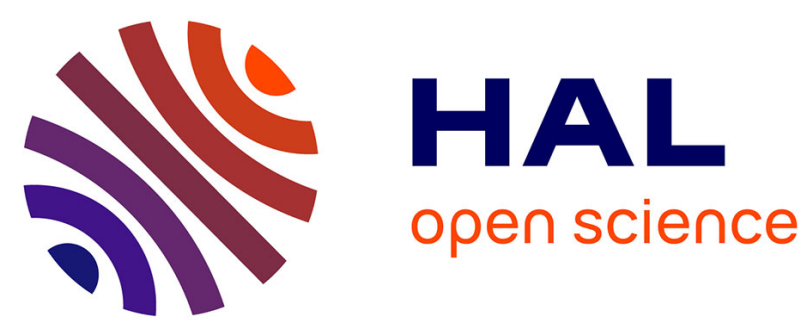

\title{
Comment organiser la délibération collégiale pour limiter ou arrêter les traitements en pédiatrie?
}

Robin Cremer, Cyril Lervat, Anne Laffargue, Justine Le Cunff, Sylvie Joriot,

Catherine Minnaert, Jean-Marie Cuisset, Karine Mention, D Thomas, Dominique Guimber, et al.

\section{To cite this version:}

Robin Cremer, Cyril Lervat, Anne Laffargue, Justine Le Cunff, Sylvie Joriot, et al.. Comment organiser la délibération collégiale pour limiter ou arrêter les traitements en pédiatrie?. Archives de Pédiatrie, 2015, 22 (11), pp.1119-1128. 10.1016/j.arcped.2015.08.004 . hal-01339001

\section{HAL Id: hal-01339001 https://hal.science/hal-01339001}

Submitted on 29 Jun 2016

HAL is a multi-disciplinary open access archive for the deposit and dissemination of scientific research documents, whether they are published or not. The documents may come from teaching and research institutions in France or abroad, or from public or private research centers.
L'archive ouverte pluridisciplinaire HAL, est destinée au dépôt et à la diffusion de documents scientifiques de niveau recherche, publiés ou non, émanant des établissements d'enseignement et de recherche français ou étrangers, des laboratoires publics ou privés. 


\title{
Comment organiser la délibération collégiale pour limiter ou arrêter les traitements en pédiatrie ?
}

\section{Organization of collaborative deliberation for limiting or withholding treatments in children}

Article à paraître dans les Archives de Pédiatrie 2015

\author{
R. Cremer ${ }^{1,2^{*}}$, C. Lervat ${ }^{3,4}$, A. Laffargue ${ }^{5}$, J. Le Cunff ${ }^{6}$, S. Joriot ${ }^{6}$, C. Minnaert ${ }^{4}$, J.-M. Cuisset ${ }^{6}$, K. Mention ${ }^{7}$, \\ D. Thomas ${ }^{4,8}$, D. Guimber ${ }^{9}$, A. Matthews ${ }^{10}$, P. Fayoux ${ }^{11}$, L. Storme ${ }^{12}$, S. Vandoolaeghe ${ }^{1}$, Pour le Groupe de \\ travail de L'EEHU de Lille ${ }^{13}$
}

1 : Espace de réflexion éthique régional. 2 rue du Professeur Laguesse, 59045, Lille

2 : Réanimation pédiatrique. Hôpital Jeanne de Flandre. CHU de Lille, 59037, Lille.

3 : Oncopédiatrie, Centre Oscar Lambret, BP 307, 59020 Lille

4 : Equipe ressource régionale en soins palliatifs pédiatriques, CHU de Lille, 59037, Lille

5 : Anesthésie pédiatrique. Hôpital Jeanne de Flandre. CHU de Lille, 59037, Lille

6 : Neurologie pédiatrique. Hôpital Roger Salengro, CHU de Lille, 59037, Lille

7 : Centre de référence des maladies héréditaires du métabolisme. Hôpital Jeanne de Flandre. CHU de Lille, 59037, Lille

8 : Maternité Jeanne de Flandre. CHU de Lille, 59037, Lille

9 : Gastroentérologie hépatologie et nutrition. Hôpital Jeanne de Flandre. CHU de Lille, 59037, Lille 10 : Pédiatrie Sociale. Hôpital Jeanne de Flandre. CHU de Lille, 59037, Lille 11 : ORL pédiatrique. Hôpital Jeanne de Flandre. CHU de Lille, 59037, Lille 12 : Réanimation néonatale. Hôpital Jeanne de Flandre. CHU de Lille, 59037, Lille 13 : Espace éthique hospitalier et universitaire (EEHU) de Lille. 2 rue du Professeur Laguesse, 59045, Lille

\section{*Auteur correspondant :}

Robin Cremer. Espace éthique hospitalier et universitaire de Lille. 2 rue du Professeur Laguesse, 59037, Lille cedex.

Mél. robin.cremer@chru-lille.fr

Tél. 03204445 02- Télécopie 0320445225 


\title{
Résumé
}

La loi du 22 avril 2005 relative aux droits des malades et à la fin de vie dite « loi Leonetti »a instauré l'obligation d'une délibération collégiale avant les décisions de limitation ou d'arrêt des traitements (LAT) pour les patients hors d'état d'exprimer leur volonté. Les modalités de cette collégialité ont été précisées par le décret du 6 février 2006 qui impose au médecin en charge du patient de prendre l'avis d'un confrère appelé à titre de consultant avant toute décision de LAT et de consulter l'équipe soignante. L'objectif de cette étude était de repérer les obstacles théoriques et pratiques à l'exercice de la collégialité et de proposer des éléments d'organisation pour les contourner.

\begin{abstract}
In 2005, the French law on patients' rights at the end of life required that decisions to withdraw or withhold life-sustaining treatments be made and carried out by the physician in charge of the patient, after obtaining advice from an independent consulting colleague and the caregiving team. The purpose of this study was to identify theoretical and practical obstacles to this collaborative deliberation and to propose practical guidelines to organize it.
\end{abstract}




\section{Introduction}

La loi du 22 avril 2005 relative aux droits des malades et à la fin de vie dite « loi Leonetti » a donné un cadre légal aux décisions de limitation et d'arrêt de traitement (LAT) et a instauré l'obligation d'une délibération collégiale avant les décisions de LAT pour les patients hors d'état d'exprimer leur volonté [1]. Les modalités de cette collégialité ont été précisées par le décret du 6 février 2006 [2] qui impose au médecin en charge du patient de prendre l'avis motivé d'un consultant avant toute décision de LAT. Ces dispositions réglementaires ont été intégrées dans l'article $37 \mathrm{du}$ code de déontologie médicale [3]. En pédiatrie, la loi fait jouer aux parents un rôle similaire à la personne de confiance chez l'adulte puisqu'il est obligatoire de les consulter avant la décision et de les informer de celle-ci avant sa mise en œuvre.

En 2012, l'espace éthique hospitalier et universitaire (EEHU) de Lille a organisé un groupe de travail pluridisciplinaire composé de professionnels prenant en charge des enfants au Centre hospitalier régional universitaire (CHRU) de Lille et de juristes de l'établissement (voir la composition en annexe 1) pour réfléchir aux modalités d'appropriation de ce texte en pédiatrie. Dans un premier temps, ce groupe a défini les situations requérant la présence d'un consultant au sens de la loi Leonetti. Il a également précisé le positionnement et le rôle théorique de ce consultant. Les résultats de ces réflexions ont été publiés dans un précédent article des Archives de Pédiatrie [4]. Dans un deuxième temps, ce groupe s'est fixé pour objectif de proposer des éléments concrets pour guider l'organisation pratique de la délibération collégiale.

\section{Méthode}

L'objectif était de traduire en pratique les principes qui avaient été définis théoriquement lors du travail précédent sur le rôle du consultant en pédiatrie [4]. Le groupe est parti du principe que c'est dans le déroulement même de la réunion, de par la manière dont elle est menée, que peut se construire le juste positionnement du consultant et l'exercice d'une délibération collégiale. Il a utilisé l'expérience acquise par les participants lors de délibérations collégiales antérieures pour décrire la réunion idéale, la définissant en quelque sorte en négatif des insatisfactions ressenties. La notion de collégialité a été prise dans son sens strictement légal, c'est-à-dire la délibération d'un collège de professionnels en charge de l'enfant, indépendamment des démarches de consultation des parents [2].

Le groupe a travaillé en trois étapes successives. Il a d'abord cherché à identifier $a$ priori les objectifs pratiques à atteindre pour qu'une réunion collégiale se déroule dans l'esprit des textes réglementaires, ainsi que les conditions favorables et défavorables à l'atteinte de ces objectifs. Dans un second temps, le groupe a proposé des solutions pratiques de contournement pour chacun des obstacles identifiés. Dans un troisième temps, le groupe a traité des questions pratiques qui avaient émergé au cours des débats, en particulier les questions de vocabulaire.

Cinq réunions de 2 heures ont été organisées de novembre 2012 à juin 2013. Chaque séance de travail a fait l'objet d'un compte-rendu qui a été discuté et validé à la séance suivante. Le texte final, écrit à partir de ces comptes-rendus, a été amendé et validé par l'ensemble des participants.

\section{Résultats}

Le produit du travail du groupe est présenté objectif par objectif. Les principales propositions sont résumées dans le tableau I.

\subsection{Réunir toutes les personnes se sentant concernées}

Pour se donner le plus de chance possible de réunir toutes les personnes se sentant concernées par l'enfant, il convient d'anticiper la réunion autant qu'il est possible et d'en annoncer la tenue dans un délai suffisamment long pour qu'elles puissent se 
libérer. Cet impératif d'anticipation avait déjà été posé dans le texte des recommandations du Groupe francophone de réanimation et d'urgence pédiatriques (GFRUP) au sujet des LAT en réanimation [5], mais une étude des pratiques en réanimation a montré que la plupart des réunions avaient été programmées du jour au lendemain [6]. La publicité (au sens premier du terme) de la réunion au sein de l'équipe soignante peut être obtenue au moyen d'un vecteur d'information habituel du service, sans qu'il y ait de recommandation particulière à proposer. En revanche, on peut se poser la question de la dénomination adéquate. Le vocable couramment employé de «réunion éthique » n'est pas à recommander puisque l'adjectif paraît qualifier la réunion elle-même et non son objet. Celui de « réunion de synthèse ", s'il est conforme à son objet, ne permet pas de différencier la réunion des autres réunions multidisciplinaires de routine. Celui de «réunion décisionnelle » n'est pas à recommander non plus puisque, en toute rigueur, c'est la délibération qui est collégiale et non la décision, laquelle est prise par le médecin en charge de l'enfant. Le groupe de travail propose d'utiliser l'expression « réunion de délibération collégiale » ou celle de « réunion collégiale ».

En toute logique, on pourrait s'attendre à ce que l'anticipation prenne toute sa pertinence pour les patients atteints d'une maladie chronique lentement progressive, dont le schéma évolutif est bien connu. Pourtant c'est précisément pour ceux-là qu'il est le plus compliqué d'anticiper. En effet, quand l'état de ces patients justifie la mise en place d'une technique de suppléance très tôt dans l'évolution de la maladie, la maintenance de cette technique et le suivi au long cours sont assurés par des équipes soignantes qui n'ont pas participé à la délibération initiale. Sous l'effet du temps, la technique devient un soin coutumier dont la prescription est un habitus ne nécessitant plus de délibération [7]. Pour ces patients, la question d'une éventuelle LAT se pose alors le plus souvent dans deux types de situations : lors d'une décompensation aigüe, ou lorsque l'on envisage un geste invasif (stomie d'alimentation, trachéotomie, pose d'un cathéter central à demeure, intervention anti- reflux, intervention de dérivation neurochirurgicale par exemple). Dans le premier cas, la difficulté réside dans la multiplicité des intervenants à réunir dans un délai court ; médecins spécialistes en charge du patient, médecin de ville, mais aussi les paramédicaux qui interviennent au domicile, les équipes de rééducation, ou les personnels des centres médico-sociaux, par exemple. Dans le second cas, quand une intervention chirurgicale est nécessaire alors qu'une limitation de certains traitements a été collégialement reconnue, la difficulté réside dans la détermination du degré de risque accepté en période péri-opératoire. A partir du moment où cette intervention chirurgicale a été jugée utile dans le cadre du projet de soins, il est naturel qu'elle se déroule dans des conditions de sécurité optimale. Pour ces enfants fragiles, cela peut paradoxalement conduire à programmer un séjour post-opératoire en réanimation qui ne serait pas jugé nécessaire pour un autre. S'il est tout à fait souhaitable d'essayer d'anticiper les complications potentielles et les limites à se donner si elles surviennent, la sécurité periopératoire optimale doit rester la règle pour un geste programmé. Comme il n'est pas raisonnable de prétendre déterminer à l'avance la durée précise de la phase post-opératoire pendant laquelle des traitements de suppléance seront appliqués en cas de défaillance d'organe ni après quelle durée ils seront interrompus, il convient d'anticiper la nécessité éventuelle d'une délibération post-opératoire. Pour le dire autrement, ces interventions ne doivent pas être insérées dans le programme de routine du bloc opératoire sans que les différents intervenants ne soient informés de l'éventualité d'avoir à décider en temps réel de ce qui relève de l'obstination déraisonnable.

\subsection{Obtenir une expression de tous}

La délibération est généralement définie comme l'action de réfléchir, d'examiner une question avant de prendre une décision. Elle suppose l'expression et la confrontation des points de vue des différents acteurs impliqués dans la situation problématique. Elle vise à trancher un problème et à permettre une décision réfléchie. La délibération peut être individuelle (réflexion d'un individu, en son for 
intérieur -(«For» vient du latin forum qui désignait, dans l'Antiquité, la place publique où se réunissaient les assemblées du peuple pour discuter des affaires publiques. Aujourd'hui, for intérieur fait référence à la conscience individuelle, à cette place délibérative en chacun de nous)-, examinant différents arguments et options possibles dans une situation donnée) ou collective. Dans le cas qui nous occupe, une délibération collégiale doit être permise. De fait, la délibération collégiale est une délibération collective, une réflexion de groupe. Il faut toutefois entendre la collégialité de manière pluri-professionnelle: collégialité ne désignant pas seulement un groupe de personnes ayant le même statut et assumant les décisions prises par la majorité de ses membres (comme le ferait, par exemple, un collège de juges), mais incluant des acteurs de soins ayant des statuts et des rôles différents [8]. C'est dans ce contexte pluridisciplinaire et pluriprofessionnel qu'obtenir l'expression de tous ceux qui interviennent dans la relation de soin avec le patient devient une exigence à l'exercice de délibération.

Si la délibération collégiale se justifie ne serait-ce qu'au regard du cadre législatif, la délibération collégiale pluriprofessionnelle est rendue légitime et nécessaire par une éthique de la discussion. Pour le philosophe et sociologue Jürgen Habermas, le langage est le paradigme de la concertation qui mène à l'action. Une communication menée rationnellement représente la seule condition pour obtenir et mener une action consentie (afin que les différents acteurs agissent librement et en toute connaissance de cause) [9]. Autrement dit, la connaissance de normes régulant l'action ne suffit pas à la décision dans une situation donnée: justifier d'une action appelle autre chose (autre que les normes) qui passe par la communication [10]. Or, cette communication comprend, pour chaque acteur, sa propre expression depuis son propre angle de vue, l'écoute d'autres expressions depuis d'autres angles de vue, puis des échanges dans le but de permettre la prise de décision. Ce qui suppose que les participants partagent un langage et un monde (un vécu) communs (cf. infra). Ces éléments conditionnent la reconnaissance nécessaire à l'expression et à l'écoute. En ce sens, cette reconnaissance est indispensable puisqu'elle confère à chaque parole la même valeur, quel que soit celui qui l'exprime, ce qu'il exprime et le temps qu'il prend pour l'exprimer. Les membres du groupe de travail ont constaté que, dans les réunions collégiales auxquelles ils avaient participé, les paramédicaux prenaient peu ou prou la parole. De manière générale, lorsqu'ils donnaient leur avis, c'était davantage en fin de réunion et pour exprimer leurs réserves. Toutefois, cette prise de parole semble favorisée par l'existence d'une expression au quotidien et non pas uniquement par une expression réservée à ce type de réunions.

Ainsi, si l'on vise une véritable réunion de délibération collégiale, veiller à la circulation et à la distribution de la parole devient un impératif dans l'organisation même de la réunion. C'est la même attention qui doit être portée à toute parole : tous les arguments doivent être entendus et considérés. Il s'agit aussi bien des arguments liés aux éléments communément appelés «objectifs » (éléments médicaux exprimés dans un langage scientifique) que des arguments liés aux éléments qualifiés de «subjectifs» (autres éléments relevant de l'enfant et de sa famille, de leur histoire, de leurs émotions, de leur environnement, etc.). $\mathrm{Si}$ les premiers sont généralement considérés comme des faits et des certitudes incontestables, les seconds ne doivent pourtant pas être réduits à des éléments interprétables non mesurables et variables. Cela confère, en effet, aux premiers éléments une primauté déterminante alors que les seconds apparaissant «en supplément », avec un impact faible et relatif. Il semble par ailleurs erroné de réserver les éléments dits subjectifs aux seuls paramédicaux comme relevant de leur domaine de compétences et exclus du domaine de compétences des médecins, comme si soigner au sens de to cure (to cure : en anglais, soigner au sens de réparer, guérir) ne concernait que les uns et soigner au sens de to care (to care : en anglais, soigner au sens de s'occuper de, se soucier de) les autres. Tout soignant, médical ou paramédical, soigne un patient et instaure, par conséquent, une relation de soin d'un sujet 
avec un autre sujet. C'est d'ailleurs pour cette raison que la parole des soignants paramédicaux compte dans la délibération au même titre que toute parole.

Pour autant, la collégialité n'implique pas l'unanimité. Et l'objectif ne consiste pas à atteindre une sorte de compromis par défaut dans lequel se dissoudraient les avis de tous. Le consensus ne s'obtient pas de manière déguisée comme si tous les participants changeaient leur positionnement initial au profit d'un positionnement moyen (au sens où il serait dans la moyenne des positionnements). Dans ce cadre, si un avis dominant émerge des échanges et si les participants en désaccord s'y rallient d'une certaine manière, c'est parce qu'ils accordent leur confiance aux autres acteurs de soins qui portent cet avis dominant et parce qu'ils envisagent, à l'issue des échanges, une légitimité à cet avis pour le patient et sa famille. Il ne s'agit donc pas d'obtenir un seul et même avis, mais de rejoindre un positionnement majoritaire tout en ayant pu exprimer son propre positionnement, celui-ci étant alors entendu au même titre que les autres. L'enjeu relève de l'implication sincère des participants dans un processus de réflexion authentiquement partagé [11] où les divergences et les désaccords ont toute leur place dans le but d'éviter tout relativisme.

Cette pratique délibérative exige donc, d'une part, une reconnaissance intersubjective, entre sujets (au sens où les sujets qui parlent ont tous la même valeur, la même dignité [12], indépendamment du rôle tenu ou du langage usité, au sens où ils ne sont pas des objets) et, d'autre part, une reconnaissance subjective du patient quel que soit son état de conscience (au sens où le patient reste toujours-déjà un sujet, à considérer comme tel dans tous les échanges délibératifs, y compris ceux qui relatent des faits étiquetés «objectifs»). Néanmoins, reconnaître les individus comme des sujets demande d'apprendre non seulement à les connaître, mais également à se connaître et se reconnaître soi-même. Le cas échéant, l'écueil serait de mettre en place une réunion artificielle, plus formelle qu'authentique.
Si la délibération collégiale représente une condition nécessaire à la décision de LAT dans ce contexte de soin, elle ne constitue pas pour autant la condition suffisante pour bien décider. Se rallier à un avis majoritaire n'altère ni ne minimise la responsabilité morale liée à la décision. Si la délibération est ici collégiale, la décision reste celle d'un seul acteur pour lequel elle doit garder un sens. Le pire écueil serait que le médecin en charge du patient et de la décision se sente précisément «déchargé », qu'il se défausse de sa responsabilité qui reste entière, voire qu'il devienne insensible.

\subsection{Eviter l'imperium scientifique}

Les soignants sont formés à la culture de la médecine basée sur les évidences, de la maitrise et du contrôle de la pathologie. Cette logique scientifique peut donner l'illusion que la pratique médicale repose sur des certitudes à considérer comme vérités absolues applicables à tous. Elle donne aussi l'illusion d'un contour précis du savoir. Cette vision conduit à nier la réalité de zones floues entre savoir et ignorance, entre les preuves scientifiques et leurs absences, à nier en réalité la zone d'incertitude. Pourtant, le savoir médical est issu de l'analyse statistique de cohortes constituées de patients choisis pour répondre de la manière la plus pertinente à une question posée sous forme d'une hypothèse à valider ou à infirmer. Il en résulte deux conséquences. La première conséquence est bien connue, mais souvent oubliée dans les périodes de situations extrêmes et de dilemme éthique: la connaissance médicale s'exprime sous forme de moyenne, pour des cohortes de patients qui sont choisis pour leur ressemblance entre eux. C'est ce qui a fait dire à Christian Mélot : «Un statisticien est un homme qui, la tête dans le four et les pieds dans la glace, dira: en moyenne je me sens bien » [13]. Les statistiques ne disent donc absolument rien du patient singulier que l'on soigne par extrapolation à partir de la connaissance de la cohorte. Il en découle que la prescription ne peut se poser sous forme d'un non-choix ou d'une évidence donnée a priori. Autrement dit, la science ne peut pas dédouaner le prescripteur de sa responsabilité. De plus, la connaissance médicale scientifique est entachée d'un risque d'erreur (par convention inférieur à 
5\%) qui est accepté quand il s'agit d'instaurer un traitement, et qui n'a pas de raison d'être refusé par principe quand on prétend rationaliser les décisions de limitation des traitements. La deuxième conséquence est moins connue. Les règles de publication scientifique et la politique éditoriale des grandes revues médicales privilégient grandement les études qui font avancer la science biologique et les études qui utilisent des méthodes d'inclusion destinées à éliminer les facteurs confondants et les points aberrants. Il s'ensuit, de manière mécanique, que plus l'étude est de haut niveau scientifique (cohorte très pure, question très pointue), plus elle emportera la conviction, mais moins elle donnera de renseignements sur le patient courant que l'on a à prendre en charge. Cette bonne appréhension de ce en quoi consiste le savoir médico-scientifique est de nature à favoriser l'acceptation par les médecins qu'il existe un degré d'incertitude et d'inconnaissable irréductible, même dans les situations scientifiques les plus balisées. Cette incertitude est la source d'une liberté qui peut être partagée collégialement, au moment de la délibération. Lorsqu'en réunion collégiale l'incertitude peut s'exprimer, les avis deviennent plus nuancés mais aussi plus partagés. Le champ des possibles est plus étendu. Des personnalités et des idées différentes se manifestent. L'incertitude est une opportunité pour mieux prendre en compte la singularité de chaque enfant et d'offrir à chacun un espace de liberté pour exprimer son opinion.

En pratique, la question de l'imperium scientifique s'est posée dès la publication du décret d'application de la loi Leonetti quand il s'est agi de définir le rôle du consultant étranger à l'équipe. La conclusion du groupe de travail précédent avait été que pour remplir un rôle conforme à l'esprit de la loi Leonetti, le consultant ne devait pas se comporter seulement en expert (c'est-à-dire uniquement faire état des connaissances scientifiques sur le problème considéré et faire l'inventaire des solutions envisageables), mais qu'il devait être un médecin à part entière, impliqué dans le choix de la conduite à tenir [4]. Il est investi d'un rôle de facilitation de la délibération, par le confère qui fait appel à lui [4].

Pour éviter l'imperium scientifique lors de la réunion de délibération collégiale, encore faut-il que le langage scientifique ne soit pas le seul employé lors de la réunion. On doit s'efforcer d'utiliser des termes simples, y compris pendant l'exposé de la situation clinique du patient, mais le problème du langage est une difficulté qui dépasse largement la question du simple obstacle à la compréhension mutuelle. En effet, si la première fonction du langage est de permettre la transmission d'informations déchiffrables, il définit de fait à la fois un champ de cohérence du raisonnement et un champ de pouvoir pour celui qui le maîtrise [14]. Qu'on le veuille ou non, la logique d'exception dans laquelle se définit le champ de la collégialité requise par la loi Leonetti induit la préséance du langage scientifique et il est difficile de s'en détacher dans la suite de la réunion. A ce problème de champ sémantique s'ajoute le problème du pouvoir et de l'autorité conférés par la maîtrise du langage « officiel». Les travaux du sociologue français Pierre Bourdieux ont montré les relations intimes et complexes entre le langage et le pouvoir. Selon cet auteur, les discours ne sont pas seulement destinés à être compris, ce sont aussi des signes d'autorité destinés à être obéis [14]. Dans le cas qui nous intéresse, l'utilisation du langage scientifique est ponctuellement une nécessité indépassable, mais il est aussi continuellement un phénomène social et institutionnel qui confère l'autorité et qui peut aboutir à une exclusion de ceux qui ne le parlent pas correctement. La situation se complique encore si on observe que les tentatives de contournement de cet obstacle peuvent avoir paradoxalement des effets contreproductifs. En effet, si l'expression des données médicoscientifiques en termes plus simples est relativement sans autre effet pervers que l'imprécision, la reformulation du discours nonscientifique depuis le langage courant vers le langage officiel au moment de la conclusion n'est pas exempte d'un risque de confiscation de la pensée, même quand la motivation de cette reformulation est authentiquement bienveillante. 


\subsection{Scénario idéal d'une délibération}

Le besoin d'une certaine scénarisation a été reconnu par les participants au groupe de travail, notamment pour différencier la réunion collégiale des autres réunions de concertations multidisciplinaires de routine. Ce scénario d'une réunion idéale a été imaginé en conformité avec les objectifs identifiés lors des discussions et en miroir des défauts constatés par les participants lors de délibérations collégiales antérieures. Il va de soi qu'il appartient aux équipes de s'en saisir pour retenir ce qui leur paraît le plus utile pour marquer cette différence. Il est possible de faire évoluer ce scénario au cours du temps, au fur et à mesure qu'une certaine habituation l'aura rendu improductive. Il ne s'agit en aucun cas d'une liturgie qui assurerait en elle-même la qualité des décisions, mais d'une représentation collective de ce que pourrait être une réunion collégiale, pensée pour respecter l'esprit de la loi Leonetti [4].

\subsubsection{Conditions matérielles}

Outre la nécessité d'une programmation «suffisamment à l'avance », la nécessité que cette réunion se tienne dans un lieu à l'écart des sollicitations cliniques de routine avait déjà été indiquée dans le texte des recommandations du GFRUP [5]. Le groupe de travail souligne qu'il conviendrait que la réunion se tienne dans une salle suffisamment vaste pour accueillir tous les participants dans des conditions égales, voire égalitaristes, de confort et de disposition, notamment en ce qui concerne les paramédicaux d'une part et le chef de service d'autre part. Il est indispensable que tous les participants participent à la totalité de la séance, ce qui implique qu'on en annonce à l'avance la durée estimée.

De plus, bien que l'esprit de transparence et le devoir de formation des plus jeunes soient de nature à autoriser l'accès de cette réunion à des professionnels de qualité et d'expérience très diverses, il n'est pas souhaitable qu'elle se déroule devant une assistance non concernée, dans une ambiance de curiosité voire de spectacle public. Pour des raisons d'efficacité, de confidentialité et de respect du malade, et pour faciliter la circulation de la parole, on peut suggérer que, exception faite du consultant, l'assistance à la réunion soit subordonnée à l'existence d'une relation de soin avec le patient dont il est question.

En raison de son rôle central dans la prise en charge du patient, c'est le médecin qui l'a en charge qui est le plus à même d'organiser la réunion collégiale et d'inviter les participants les plus adéquats : les soignants (médicaux et paramédicaux), le consultant, mais également les psychologues ou assistantes sociales qui détiennent des informations contextuelles déterminantes.

3.4.2 Direction de la réunion, annonce du déroulement, rappel des questions posées

Dans ce contexte, c'est le médecin en charge du malade qui prend l'avis de l'équipe paramédicale et de ses collègues et fait appel à un confrère étranger à l'équipe en tant que consultant obligatoire, avant de prendre luimême une décision dont il sera le responsable légal. Il est donc logique qu'il introduise la réunion et qu'il la conclue. Il est nécessaire qu'il explique à chaque fois ce contexte particulier dès le début de la réunion.

Il est également indispensable qu'il présente le rôle des différents participants (chef de service, médecin de ville, médecin spécialiste hospitalier qui suit l'enfant en routine, médecin expert de la maladie, médecin consultant au sens de la loi, paramédicaux en charge de l'enfant). Les situations de cumul de plusieurs rôles ne sont pas des obstacles à la collégialité [4], mais il est utile qu'elles soient clairement identifiées. On ne saurait trop insister sur la nécessité de poser explicitement, dès le début de la réunion, lequel des participants est investi du rôle de médecin consultant et d'en expliciter le rôle à chaque fois [4].

Il est souhaitable que le cadre légal et les questions posées à la collégialité soient annoncés en début de réunion de manière à ce que les participants respectent un plan de raisonnement commun. D'ailleurs, la première question à laquelle la collégialité devra répondre de manière explicite est celle du 
cadre : le patient est-il bien dans une situation qui peut être assimilée à "un état avancé ou terminal d'une affection grave et incurable»? En effet, bien que cette expression puisse paraître inadaptée à la pédiatrie et tout particulièrement à la néonatalogie, elle constitue la condition légale qui valide le recours à la collégialité (Art. L. 1111-13 du code de santé publique). A l'époque de la promulgation de la loi Leonetti, les réanimateurs pédiatres avaient assimilé cette formulation à un constat de fin de vie et ils avaient souligné qu'elle correspondait à la situation clinique de seulement la moitié des décisions de LAT [15]. A l'occasion de l'affaire Lambert, le Conseil d'Etat a clairement confirmé qu'il ne s'agit pas de savoir si le patient est ou non en fin de vie [16], mais de constater, dans une optique beaucoup plus large, l'absence de ressource thérapeutique pour améliorer l'évolution d'une affection dont le pronostic est mauvais. A ce sujet, les commentaires de l'article 37 du code de déontologie indiquent que «c'est une appréciation globale de la cinétique de la maladie et une évaluation des possibilités d'amélioration qui doivent être recherchées » [3]. En pédiatrie et en néonatalogie cela nécessite une projection à bien plus long terme qu'en médecine d'adulte, mais c'est un exercice que les pédiatres sont habitués à faire et à partager avec les parents. En théorie, cette discussion ne devrait pas entraîner la collégialité dans de longues discussions médico-scientifiques puisque la majorité des examens complémentaires destinés à fonder le diagnostic et le pronostic auront été réalisés et interprétés avant la réunion. En principe, les avis d'experts auront été obtenus au préalable.

La deuxième question est : il y a-t-il des actes indiqués du fait de l'état du patient qui peuvent contrevenir à l'interdiction de l'obstination déraisonnable ? Ces actes ont été définis comme ceux qui sont «inutiles, disproportionnés ou n'ayant d'autre effet que le seul maintien artificiel de la vie » (Art. L. 111113 du Code de Santé Publique).

En cas de réponse positive, c'est seulement dans un troisième temps que se pose alors la question des modalités pratiques de mise en œuvre des LAT et de la conception d'un nouveau projet de soins, adapté au deux constats précédents, et acceptable par la famille et par l'équipe soignante. Si on ne peut soulager la souffrance du patient, dans le cadre d'un nouveau projet de soins à forte dimension palliative, alors on peut avoir à envisager l'emploi d'un traitement "ayant pour effet secondaire d'abréger sa vie» (article 1110-5 du Code de Santé Publique).

\subsubsection{Déroulement}

Le déroulement proposé par le groupe est résumé dans le tableau II. L'exposé de l'état médical et psychologique du patient doit nécessairement constituer le premier temps de la réunion et nous avons indiqué plus haut les inconvénients de cet état de fait incontournable. Il a semblé au groupe de travail que la participation de paramédicaux à cet exposé pouvait limiter la portée de ces inconvénients dans la suite de la délibération. On peut en attendre une meilleure intelligibilité pour les non médecins. Il va de soi que cette participation doit avoir été préparée en collaboration avec le médecin en charge du patient, faute de quoi on ferait courir aux paramédicaux un risque inacceptable de discrédit. La présentation des autres éléments constitutifs de la personne du patient (son développement, sa personnalité, son acceptation plus ou moins difficile des traitements), de sa famille, de son entourage et de son milieu est également un des points importants de cet exposé initial. Il est souhaitable qu'elle soit faite à ce stade de la réunion puisqu'il s'agit d'éléments indispensables à la détermination du caractère proportionné ou non des traitements. Là aussi, la participation des paramédicaux est à favoriser, d'autant plus qu'ils sont souvent détenteurs d'informations qui ne figurent pas au dossier médical qui leur ont été livrées dans un climat de confiance.

Un avantage de l'adoption d'un plan de discussion préétabli est qu'il donne des repères pour la distribution de la parole tout au long de la discussion. On peut suggérer qu'un tour de table soit organisé à chaque étape, de manière à ce que chaque catégorie d'intervenants prenne la parole sur chacun des points. On peut 
également proposer que ceux qui ont habituellement le plus de difficulté à s'exprimer dans un groupe le fasse en début de chacun des tours de table, pour limiter autant que faire se peut les phénomènes d'autocensure potentiellement induits par la difficulté de parler après un membre influent de l'équipe.

Le rôle du médecin consultant au sens de la loi Leonetti n'a pas vocation à être particulièrement mis en avant, mais il convient de rappeler que sa réponse explicite à chacune des questions est nécessaire pour que son intervention ne se limite pas à un rôle d'expert détaché de la décision [4].

La rédaction des conclusions de la délibération dans le dossier médical est le dernier temps de la réunion et devrait être systématiquement faite en temps réel afin que ses termes soient choisis collégialement. Ces conclusions doivent être explicites, c'est-à-dire intelligibles pour des lecteurs étrangers à l'équipe, notamment pour les autorités judiciaires. En effet, les équipes soignantes utilisent fréquemment des expressions qui leur sont propres pour désigner différentes stratégies de prise en charge en fonction de l'importance de leur composante palliative («soins proportionnés », "soins adaptés », «soins de confort », «non escalade », par exemple). Si ces expressions peuvent avoir un sens très fort et très précis au sein de ces équipes, il faut reconnaître que ce n'est pas toujours le cas et qu'elles peuvent être interprétées de manière variable par un tiers. Les décisions de ne pas transférer en réanimation, de ne pas entreprendre de réanimation en cas d'arrêt cardiaque, de ne pas instituer de nouveau traitement en cas d'aggravation, ou d'interrompre tel ou tel traitement doivent figurer en clair. De plus, on ne peut que recommander l'utilisation des expressions issues de la loi elle-même dans la rédaction des motivations, notamment le constat d'un état avancé ou terminal d'une affection grave et incurable et la notion d'obstination déraisonnable.

\subsubsection{Désaccords avec les parents}

Laisser aux parents la possibilité de choisir leur niveau d'implication dans les décisions de LAT [5] revient à accepter que la décision proposée après délibération collégiale puisse ne pas leur convenir. Dans la pratique, ces oppositions sont rares. Ainsi, dans une étude française publiée en 2011 portant sur plus de 5000 séjours en réanimation pédiatrique et plus de 300 processus de LAT, les parents se forgeaient une opinion en 48 heures. Un tiers des parents considérait que la décision revenait au médecin ou n'exprimait pas d'avis. Leurs réactions à l'annonce de la décision étaient $2 / 3$ d'approbations et $1 / 3$ de résignations. Les auteurs n'ont enregistré qu'une seule opposition des deux parents à l'arrêt du traitement et une opposition d'un parent à la poursuite des traitements alors que l'autre n'exprimait rien [6].

On ne saurait trop recommander de consigner dans le dossier médical la réaction des parents et d'éventuelles contre-propositions faites par l'équipe soignante. En cas de désaccord, le fait qu'il soit expressément noté que la poursuite de certains traitements a eu lieu à la demande des parents et contre l'avis de la collégialité est de nature à protéger les équipes de l'accusation d'obstination déraisonnable. Elle peut également être un facteur de réappropriation du projet de soin par les paramédicaux.

\section{Discussion}

Paradoxalement, alors les évolutions futures de la législation sur la fin de vie devraient accroitre les occasions de recours à la collégialité [17], il n'existe aucune publication médicale explicitant ce terme et le traduisant en termes pratiques, dans l'objectif d'une appropriation par les équipes. S'il est aisé de comprendre qu'il s'agit de ne pas laisser un médecin décider seul, le caractère multiprofessionnel de la collégialité en rend la pratique difficile dans le milieu hiérarchisé de l'hôpital. De plus, le caractère fondamental du savoir scientifique en médecine donne de fait un caractère dominant au langage de la science qui complique l'organisation d'une réelle discussion argumentative.

Ce texte est la synthèse d'une série de réunions ayant impliqué des représentants de la 
quasi-totalité des professionnels de l'enfant amenés à travailler ensemble dans un même site. Il ne s'agit pas de recommandations directement généralisables, mais d'un travail local que d'autres équipes pourraient s'approprier. Il pourrait également être utilisé, parmi d'autres, si de véritables recommandations de société savante devaient élaborées.

Le groupe a employé la méthode qualitative la plus simple compte tenu du nombre de participants et de leur communauté d'exercice : la discussion argumentative suivie de la rédaction en commun d'un texte consensuel. Pour cette raison, l'argumentation qui a conduit les rédacteurs à adopter ces points de vue figure dans les résultats de l'étude au même titre que les recommandations qu'ils en ont tiré pour eux-mêmes. C'est cette argumentation qui permettra à d'autres de s'en saisir (ou non) pour les adapter à leur propre environnement de travail.

\section{Conclusion}

La collégialité est un exercice difficile dans un milieu très hiérarchisé où le savoir scientifique est nécessairement une valeur fondamentale. Les deux principaux obstacles identifiés par le groupe de travail sont la banalisation des procédures qui est source de déresponsabilisation (la réunion collégiale deviendrait une réunion comme les autres), et la question du pouvoir conféré par la maîtrise du langage médico-scientifique (excluant de fait ceux qui ne le parlent pas ou qui ne sont pas reconnus pour le parler).

Il revient à chaque équipe de s'approprier la démarche proposée, afin de concevoir un scénario pensé pour contourner ces deux écueils. Cet effort peut être de nature à améliorer la collaboration des différents acteurs de soin autour d'un projet formulé de manière positive.

\section{Références}

1. Loi $\mathrm{n}^{\circ}$ 2005-370 du 22 avril 2005 relative aux droits des malades et à la fin de vie.
Journal Officiel de la République Française du 23 avril 2005 2005:7089.

2. Décret $\mathrm{n}^{\circ}$ 2006-120 du 6 février 2006 relatif à la procédure collégiale prévue par la loi $\mathrm{N}^{\circ}$ 2005-370 du 22 avril 2005 relative aux droits des malades et à la fin de vie et modifiant le code de la santé publique. Journal Officiel de la République Française du 7 février 2006 2006; $\mathrm{N}^{\circ} 32: 1974$.

3. Conseil de l'Ordre des médecins. Commentaires sur l'article 37 du code de déontologie médicale. Disponible en ligne sur http://wwwconseil-

nationalmedecinfr/article/article-37-

soulagement-des-souffrances-limitation-ou-

arret-des-traitements-261;consulté en mars 2011.

4. Cremer R, Fayoux P, Guimber D, et al. Le médecin consultant pour les limitations et les arrêts de traitement en pédiatrie. Arch Pédiatr 2012;19:856-62.

5. Hubert $\mathrm{P}$, Canoui $\mathrm{P}$, Cremer $\mathrm{R}$, et al. Limitations et arrêts des traitements actifs en réanimation pédiatrique : recommandations du GFRUP. Arch Pédiatr 2005;12:1501-8.

6. Cremer R, Hubert P, Grandbastien B, et al. Prevalence of questioning regarding lifesustaining treatment and time utilisation by forgoing treatment in francophone PICUs. Intensive Care Medicine 2011;37:1648-55.

7. Pordès C. Nutrition entérale à domicile et trajectoires difficiles en pédiatrie : étude qualitative des représentations des questionnements et décisions chez les soignants. Thèse Med Lille 22011.

8. Vinatier I, Fiancette M, Lebert C, et al. Collégialité dans les décisions de limitation ou d'arrêt de traitement en réanimation. Réanimation 2014;23:503-10.

9. Habermas J. Théorie de l'agir communicationnel. Paris: Fayard; 1987.

10. Habermas J. De l'éthique de la discussion. Paris: Cerf; 1992.

11. Haddad L. Fonctionnement de la collégialité. In: Puybasset L, editor. Enjeux éthiques en réanimation. Paris: Springer Verlag; 2010. p. 275-86.

12. Auteur de la notice. Dignité. In: CantoSperber M, editor. Dictionnaire d'éthique et de 
philosophie morale (tome 1). Paris: QuadrigePUF Edition; 1996. p. 523-8.

13. Mélot C. Vous avez dit statistiquement significatif ? XXXIV ${ }^{\circ}$ Congrès de la société de réanimation de langue française, Paris 2008:disponible en ligne sur http://www.srlf.org/data/FlashConfs/2008/3213 I.

14. Bourdieux P. Ce que parler veut dire. Paris: Fayard; 1982.

15. Cremer R, Hubert $\mathrm{P}$, Dauger $\mathrm{S}$, et al. Limitations et arrêt des traitements en réanimation pédiatrique : résultats d'une étude multicentrique de 18 mois (Résumé). Réanimation 2009;18 Suppl:115-6.
16. Conseil d'Etat. Assemblée du 14 février 2014, Mme Lambert et autres, requêtes numéro 375081, 375090, 375091. 2014.

17. Leonetti J, Claeys A. Rapport de présentation et texte de la proposition de loi e MM. Alain Claeys et Jean Leonetti créant de nouveaux droits en faveurs des malades et des personnes en fin de vie. La documentation française 2015:accessible en ligne sur http://www.ladocumentationfrancaise.fr/rapport s-publics/144000752/.

Conflits d'intérêts : aucun

Tableau I :

Objectif

\section{Propositions du groupe de travail}

Obtenir une expression de tous

Réunir toutes les personnes concernées
Limiter l'imperium scientifique
- Anticiper la réunion et l'annoncer.

- Utiliser un terme qui identifie le caractère particulier de la réunion et qui la différencie des autres staffs et réunions multidisciplinaires.

- Annoncer un horaire précis et une durée prévisible.

- Organiser la réunion dans un lieu susceptible d'accueillir toutes les personnes invitées dans des conditions égales de confort, à l'écart des sollicitations des services.

- Présenter les différents participants et expliciter leur rôle par rapport au patient (médecin traitant habituel, médecin en charge du patient, interne en charge du patient, médecin spécialiste, infirmières, cadres de santé, psychologue, assistante sociale, etc.).

- Annoncer quel(s) médecin(s) est (sont) investi(s) du rôle de consultant au sens de la loi Leonetti.

- Rappeler le contexte spécifique de la réunion et le rôle de chacun comme force de délibération avant la décision.

- Impliquer des soignants non médecins dans la présentation du cas.

- Utiliser un plan de discussion préétabli, question par question, prévoyant l'expression de chacun à chaque étape.

- Exposer les éléments de contexte du patient (famille, contexte social et psychologique, acceptabilité des traitements) à la suite des éléments cliniques, lors d'un même temps de présentation du dossier.

- Impliquer des soignants non médecins dans la présentation du cas étudié.

- Utiliser un vocabulaire simple dans la présentation des données cliniques et éviter autant que possible le jargon médico-scientifique.

- Eviter de reformuler les éléments de discussions apportés par les non médecins dans un langage exclusivement scientifique.

- Obtenir du consultant qu'il ne se comporte pas en expert détaché de la décision à prendre, mais qu'il donne son avis sur celle-ci et qu'il le motive. 
Tableau II : plan de la réunion et rôles de chacun

Tour de table 1

- Demandes de précisions, questions, remarques, informations complémentaires.

L'enfant est-il dans une situation assimilable à un "état avancé ou terminal d'une affection grave et incurable »?

- C'est une appréciation globale de la cinétique de la maladie et une évaluation des possibilités d'amélioration qui doivent être recherchées [3].

- Il ne s'agit pas diagnostiquer un état de fin de vie [16].

\section{Tour de Table 2}

- Demandes de précisions, questions, remarques, réponse à la question.

Il y a-t-il des actes indiqués du fait de l'état du patient qui peuvent contrevenir à

l'interdiction de l'obstination déraisonnable?

- $\quad$ soins « inutiles, disproportionnés ou n'ayant d'autre effet que le seul maintien artificiel de la vie » (Art. L. 1111-13 du Code de santé publique).

Tour de Table 3

- Demandes de précisions, questions, remarques, réponse à la question

Modalités pratiques de mise en œuvre des LAT et conception d'un nouveau projet de

- Si on ne peut soulager la souffrance du patient, dans le cadre d'un nouveau projet de soins à forte dimension palliative, alors on peut avoir à envisager l'emploi d'un traitement « ayant pour effet secondaire d'abréger sa vie » (article 1110-5 du Code de Santé Publique).

Tour de Table 4

- Demandes de précisions, questions, remarques, avis différent, accord ou désaccord

Conclusion

- Rédaction des conclusions de la réunion et motivations de celle-ci.

Intervenant(s)

Médecin sénior en charge du patient

Médecin (ou interne) en charge du patient et des membres de l'équipe paramédicale

Tous

Médecins en charge du patient, experts, consultant

Tous

Tous

Tous

Tous

Tous

Médecin en charge du patient et consultant 
Annexe 1 : Participants au groupe de travail

Robin CREMER, réanimateur pédiatre, CHU de Lille et coordonnateur adjoint de l'EEHU de Lille

Jean-Marie CUISSET, neurologue pédiatre, CHU de Lille

Pierre FAYOUX, chirurgien ORL pédiatrique, CHU de Lille

Marie-Bénédicte GLACET, puéricultrice, CHU de Lille

Dominique GUIMBER, gastro-entérologue pédiatre, CHU de Lille

Justine LE CUNFF, psychologue, CHU de Lille

Catherine LE GRAND-SEBILLE, socioanthropologue, Université de Lille 2

Sylvie JORIOT, neurologue pédiatre, CHU de Lille

Nathalie JUDAS, psychologue, CHU de Lille

Anne LAFFARGUE, anesthésiste pédiatrique, CHU de Lille

Cyril LERVAT, oncopédiatre, Centre Oscar Lambret, Lille et Equipe régionale ressource en soins palliatifs pédiatriques, $\mathrm{CHU}$ de Lille
Catherine MINNAERT, infirmière, Equipe régionale ressource en soins palliatifs pédiatriques, $\mathrm{CHU}$ de Lille

Anne MATTHEWS, pédiatre, pédiatrie sociale, CHU de Lille

Karine MENTION, pédiatre, maladies métaboliques, CHU de Lille

Brigitte NELKEN, hématopédiatre, CHU de Lille

Rony SFEIR, chirurgien pédiatrique, $\mathrm{CHU}$ de Lille

Laurent STORME, pédiatre en réanimation néonatale, CHU de Lille

Dominique THOMAS, pédiatre en maternité et à l'Equipe régionale ressource en soins palliatifs pédiatriques, $\mathrm{CHU}$ de Lille

Caroline THUMERELLE, pneumologue pédiatre, CHU de Lille

Audrey VASSEUR, psychologue, CHU de Lille

Sylvie VANDOOLAEGHE, chargée de développement, coordinatrice adjointe de l'EEHU de Lille 